\title{
Energia Wavelet aplicada à análise da Corrente de Partida de Motores para o Diagnóstico de Falhas
}

\author{
Matheus A. G. Calsavara*. Lane M. R. Baccarini*. Paulo C. M. Lamim Filho* \\ Fabiano B. Bastista**
}

\author{
* Departamento de Engenharia Elétrica, Universidade Federal de São João Del Rei, \\ São João del-Rei, Minas Gerais, CEP: 36307-352 (e-mail: matheus.guilarducci@ hotmail.com, \\ rabelo@ufsj.edu.br,lamim@ufsj.edu.br). \\ ** Departamento de Engenharia Mecânica, Universidade Federal de São João Del Rei, \\ São João del-Rei, Minas Gerais, CEP: 36307-352 (e-mail: fabianchini@ufsj.edu.br).
}

\begin{abstract}
The main objective of this article is propose a method for detecting broken bars faults in a three-phase induction motors through analysis of the starting current. Were used signal processing and analysis well-known techniques, such as Hilbert Transform and Wavelet Transform, adding the concept of Wavelet Energy. In order to establish a more robust and effective analysis, simulations were made for different operating conditions and broken bars. The methodology was also validated with experimental data obtained in a test bench in a $3 \mathrm{CV}$ motor, which demonstrated the robustness of the proposed technique.
\end{abstract}

Resumo: O objetivo principal desse trabalho é propor um método para detecção de falhas de barras quebradas em motores de indução trifásicos através da análise da corrente de partida. Foram utilizadas técnicas bastantes conhecidas para processamento e análise de sinais, como a Transformada de Hilbert e a Transformada de Wavelet, acrescentando o conceito de Energia da Wavelet. Com intuito de estabelecer uma análise mais robusta do método proposto foram realizadas simulações para diferentes condições de operação e barras quebradas. A metodologia também foi validada com dados experimentais obtidos numa banca de teste em um motor de $3 \mathrm{CV}$, o que demonstrou a robustez da técnica proposta.

Keywords: Broken Bars; Wavelet Transform; Applications in Industry; Energy Wavelet; Fault Detection

Palavras-chaves: Barras Quebradas; Transformada de Wavelet; Aplicações na Indústria; Energia da Wavelet; Deteç̧ão de Falhas.

\section{INTRODUÇÃO}

Os acionamentos elétricos durante muitos anos foram dominados pelas máquinas de corrente contínua. Essa realidade acabou sofrendo mudanças com o surgimento dos motores de indução, que são máquinas elétricas robustas e adaptáveis a uma vasta gama de aplicações. Por esse motivo, no setor industrial os MITs correspondem a cerca de $95 \%$ do total de motores instalados (Massirer, 2007) e são objetos de grande parte dos estudos relacionados a forma de evitar e corrigir falhas que podem ocorrer em conjunto com o envelhecimento natural da máquina.

As plantas industriais são dependentes do funcionamento dos motores, o que faz com que a confiabilidade desses equipamentos se torne um ponto crítico devido ao alto custo operacional de uma parada no processo produtivo da empresa, os quais podem representar de $15 \%$ a $40 \%$ do custo total de muitos produtos (Avelar et al., 2011). Mesmo considerando o baixo custo dos motores de indução comparado as demais máquinas rotativas, não é interessante economicamente manter máquinas sobressalentes, o que demonstra a importância de um monitoramento constante do motor para garantir uma operação segura e a qualidade na produção (Bacarinni, 2010).

No meio acadêmico foram propostas diversas técnicas para detectar e analisar falhas nas máquinas elétricas, porém muitas ainda são invasivas e nem sempre apropriadas para o ambiente industrial.

De acordo com (Zhang et al., 2011), 44\% das falhas ocorrem nos rolamentos da máquina, $26 \%$ corresponde a curtos circuitos nos enrolamentos do estator, $8 \%$ estão relacionadas a falhas nas barras do rotor, e os outros $22 \%$ correspondem as demais falhas que podem ocorrer, como desequilíbrio entre as tensões de alimentação do motor, harmônicos, afundamento de tensão, etc. Apesar de representar um percentual pequeno, a quebra de barra do rotor pode causar danos irreversíveis ao motor, o que justifica o desenvolvimento de algoritmos para o diagnóstico desta falha.

Os métodos tradicionais baseados na análise espectral (Fourier) aplicados para detecção de falhas de barras quebradas em regime permanente tornam-se inadequados para condições de baixo escorregamento ou de variação de velocidade (Nobrega Sobrinho et al., 2018). Nessas situações, 
a frequência de banda lateral aproxima-se do valor da frequência fundamental ou varia conforme a velocidade da máquina.

Alguns trabalhos recentes, tais como: Sridhar, Rao e Jade (2015); Hernández et al. (2015); Bidgoli e Poshtan (2017), propõem o uso da Transformada de Wavelet para detecção de barras quebradas em regime permanente, enquanto, Ahamed et al. (2010) e Riera-Guasp et al. (2012) apresentaram métodos utilizando os sinais da máquina durante o regime transitório.

Pineda-Sanches et al. (2011) apresentou um método em que um sinal estacionário é criado a partir da Transformada de Fase-Polinomial de um sinal transitório. Este novo sinal contém as informações sobre os componentes da falha ao longo dos diferentes regimes abrangidos pelo transiente, podendo ser analisado posteriormente pela Transformada de Fourier.

Os métodos que utilizam a assinatura de corrente transitória do motor são técnicas desenvolvidas recentemente para diagnóstico de motores de indução (Pineda-Sanches et al., 2011). O método proposto neste trabalho utiliza ferramentas consolidadas para análise e processamento de sinais, permitindo explorar a corrente de partida do motor para extrair as informações relacionadas as falhas de barras quebradas, com a vantagem de aplicação em máquinas que operam durante intervalos de tempo curtos e/ou com baixa carga, resultando em baixas condições de escorregamento.

Com base no modelo assimétrico proposto por Baccarini (2005) é possível simular o funcionamento do MIT diante da presença de várias condições de barras quebradas. Esta etapa inicial permitiu o desenvolvimento da técnica e a validação em motores de diferentes características. Após esta etapa, a técnica proposta foi validada numa bancada de teste.

\section{METODOLOGIA}

Utilizou-se para as simulações computacionais motores de 3 CV e 500 HP, sendo que os parâmetros do MIT com potência nominal de $3 \mathrm{CV}$ foram encontrados através de ensaios realizados no motor real presente na bancada de teste e também utilizado para os testes experimentais. Os parâmetros do MIT de 500 HP estão descritos em (Krause et al. 2002, p. 165). O modelo dinâmico da máquina foi implementado no software $M A T L A B^{\circledR}$. As equações diferenciais foram resolvidas pelo método de integração Runge Kutta de $4^{\mathrm{a}}$ ordem com um passo de integração igual a $10^{-5} \mathrm{~s}$.

As equações que descrevem o modelo assimétrico do Motor de Indução Trifásico podem ser obtidas em (Baccarini, 2005). As Transformadas de Wavelet e de Hilbert são técnicas bastante conhecidas. Portanto, não serão descritas neste trabalho.

\subsection{Energia da Wavelet - WE}

Assim como na Transformada de Fourier, existe uma metodologia análoga para o cálculo de energia na Transformada de Wavelet. Os autores Liu e Huang (2005) observaram que existe uma relação entre a energia do sinal obtido pela WT e o sinal original. Diante disso, eles introduziram o conceito de Energia da Wavelet (Wavelet Energy - WE), que determina a energia total de um sinal pela soma dos quadrados de seus coeficientes de aproximação e detalhe. A energia em cada banda de frequência para o nível de decomposição $\mathrm{c}=1,2, \ldots, 2 \mathrm{j}$ é definida pela seguinte equação:

$$
\mathrm{E}_{\mathrm{c}}=\left|\mathrm{b}_{\mathrm{j}, \mathrm{c}}(\mathrm{t})\right|^{2}
$$

Sendo que bj,c(t) consiste na amplitude do coeficiente da Transformada Discreta de Wavelet para cada ponto discreto em sua respectiva banda de frequência.

A energia total do sinal é dada por:

$$
\mathrm{E}=\left(\sum_{\mathrm{c}=1}^{2^{\mathrm{j}}} \mathrm{E}_{\mathrm{c}}\right)
$$

Pela normalização dos valores de energia, obtém-se a WE, que corresponde ao nível de energia em cada pacote Wavelet. Dessa forma, através da Equação 3 obtém-se a energia normalizada em cada banda de frequência.

$$
\mathrm{H}=\left[\frac{\mathrm{E}_{1}}{\mathrm{E}}, \frac{\mathrm{E}_{2}}{\mathrm{E}}, \ldots, \frac{\mathrm{E}_{2^{\mathrm{j}}}}{\mathrm{E}}\right]
$$

Diante da ocorrência de assimetrias no rotor de uma máquina de indução, a assinatura da falha está contida em determinada banda de frequência. Nesse caso, de acordo com o conceito de WE, pode-se calcular o nível de energia em cada banda e, conforme sua variação, pode-se detectar a presença da falha. Isso torna a WE um excelente parâmetro quantitativo para identificar e caracterizar fenômenos de interesse, tal como a detecção de defeitos na máquina (Alves, 2017).

\section{RESULTADOS}

As análises e discussões apresentadas nessa seção foram obtidas através de simulações computacionais e testes experimentais. Para os testes experimentais utilizou-se um motor de indução da marca WEG, com potência de $3 \mathrm{CV}, 4$ polos, $220 \mathrm{~V}, 60 \mathrm{~Hz}, 8.7$ A e 1730 RPM. Um motor de corrente contínua operando como gerador foi utilizado como carga, sendo conectado ao motor principal através de um acoplamento flexível com um torquímetro, como está demonstrado na Figura 1. Além disso, foram utilizados dois rotores idênticos, diferenciando-os apenas em relação ao fato de um deles possuir uma barra quebrada e o outro não.

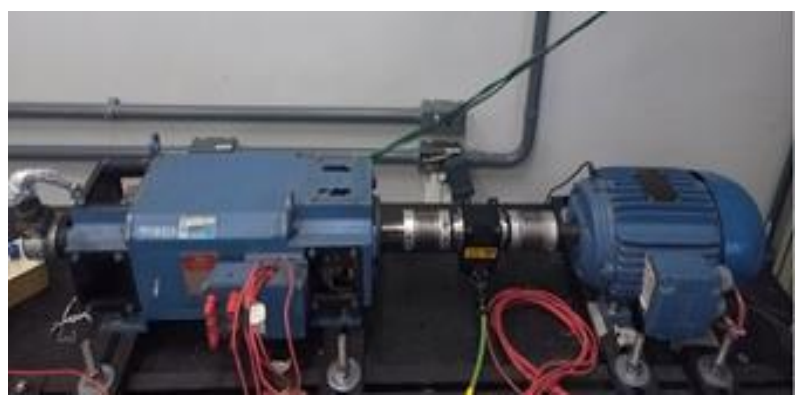

Figura 1 - Bancada experimental utilizada para os testes 
A aquisição dos sinais de corrente foi realizada com uma frequência de amostragem de $10.24 \mathrm{kHz}$ durante todo o período transitório de partida do motor. Para os testes experimentais foi utilizado um sensor de efeito Hall A622 AC/DC - $100 \mathrm{~Hz}$ Tectronix, conectado a uma placa de aquisição NI PCI-4461 da National Instruments e submetido a um filtro anti-aliasing com frequência de corte de $2.5 \mathrm{kHz}$. Os sinais foram coletados com motor operando sem carga no eixo para demonstrar os benefícios da técnica apresentada em relação as metodologias que utilizam os sinais em regime permanente.

A Transformada de Hilbert utilizada para extrair o envelope do sinal de corrente é uma técnica de análise de sinal bem conhecida e amplamente utilizada na identificação de falhas no motor de indução. O envelope obtido do sinal transiente possui as informações relacionadas à falha, e por isso, foi utilizado em conjunto com a Transformada de Wavelet. A Figura 2 apresenta o envelope da corrente de partida do motor obtido a partir do sinal de corrente amostrado nos testes experimentais para o rotor na condição de uma barra quebrada.

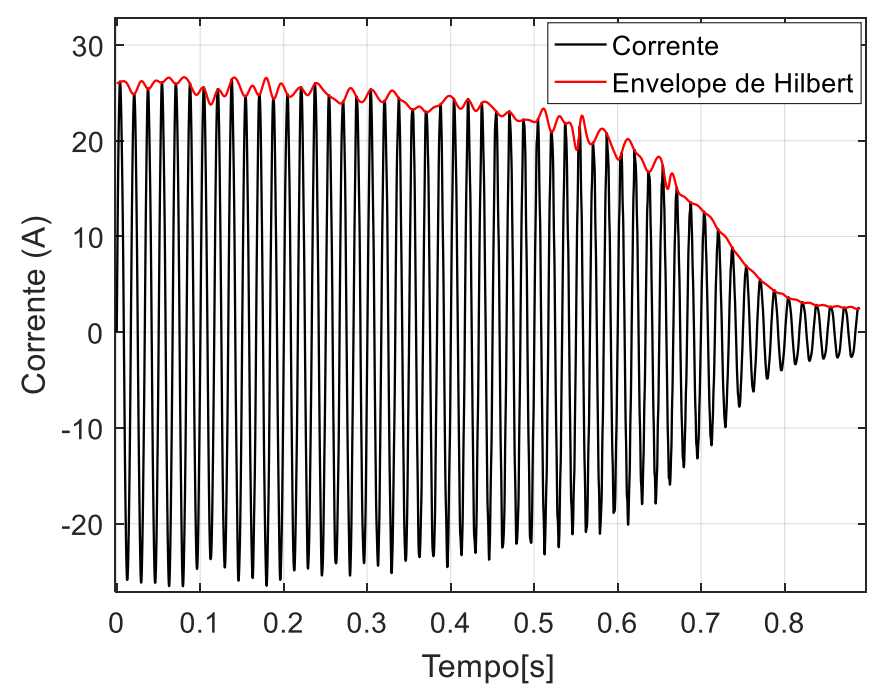

Figura 3 - Corrente do estator com quebra de uma barra do rotor (curva preta) e envelope de Hilbert (curva vermelha) -

(Resultado experimental)

Para análise do envelope nas diferentes condições foi utilizada a ferramenta Transformada Discreta de Wavelet (DWT). Várias Wavelets mães foram testadas, porém o melhor resultado se deu com o uso da Daubechies-10 (db10). A decomposição do sinal em intervalos de frequência, utilizado para as análises em regime transitório, foi feita até o nível 9, considerando que as faixas de frequência são funções da frequência de amostragem, conforme a Equação 4.

$$
n=\operatorname{int}\left(\frac{\log \left(f_{s} / F_{d}\right)}{\log (2)}\right)+2
$$

Onde, Fd é a frequência de amostragem e fs a frequência fundamental. As faixas de frequência decompostas para cada coeficiente de detalhe são mostradas na Tabela 1. Os níveis 10 e 11 correspondem a análise realizada em regime permanente. Devido a extração da frequência fundamental no sinal de corrente, a frequência de barra quebrada assume um valor consideravelmente baixo, sendo necessária a realização da decomposição em mais níveis, conforme a indicação feita através da Equação 4.

Tabela 1 - Faixas de Frequências para cada Nível de Decomposição considerando DWT

\begin{tabular}{|c|c|}
\hline Nível & Banda de Frequência (Hz) \\
\hline D1 & $2560-5120$ \\
\hline D2 & $1280-2560$ \\
\hline D3 & $640-1280$ \\
\hline D4 & $320-640$ \\
\hline D5 & $160-320$ \\
\hline D6 & $80-160$ \\
\hline D7 & $40-80$ \\
\hline D8 & $20-40$ \\
\hline D9 & $10-20$ \\
\hline D10 & $5-10$ \\
\hline D11 & $2,5-10$ \\
\hline
\end{tabular}

A detecção da falha pode ser visualizada através das oscilações presentes nos coeficientes da Wavelet e representada através da variação nos níveis de energia. Sabese que a falha de barra quebrada no rotor aparece nas bandas laterais da frequência fundamental da máquina, conforme a equação 5. Devido a aplicação da Transformada de Hilbert, a componente fundamental do sinal é filtrada, e com isso, os harmônicos referentes a falha aparecem em novas bandas de frequência. Por isso considerou-se apenas os três últimos níveis de decomposição da Wavelet (D7, D8 e D9) para caracterizar a presença da falha.

$$
f_{b q}=\left|(1+2 k s) f_{s}\right|
$$

Em que, $\mathrm{f}_{\mathrm{bq}}$ corresponde a frequência da banda lateral; s é o escorregamento da máquina; $\mathrm{f}_{\mathrm{s}}$ é a frequência fundamental de alimentação e k representa um número inteiro.

Os valores de níveis de energia encontrados para cada condição quantificam as variações nas oscilações e demonstram a influência da falha sobre o sinal de corrente analisado. Isso torna o conceito de Energia da Wavelet (WE) um excelente parâmetro quantitativo para identificar e dimensionar os fenômenos de interesse, tal como a detecção de falhas da máquina (Alves, 2017).

\subsection{Resultados das Simulações}

\section{A. Motor de $3 \mathrm{CV}$}

Através de ensaios de rotor travado e de rotor livre foi obtido os parâmetros do modelo matemático do motor de $3 \mathrm{CV}$ da bancada. Com a simulação computacional, além da condição inicial de falha (uma barra quebrada), pode-se simular a existência de mais barras quebradas no rotor. As Figuras 4 e 5 mostram a análise da falha no rotor através dos coeficientes da Wavelet para condição de rotor saudável comparando com uma e duas barras quebradas, respectivamente. Os níveis de energia para as três condições de operação do motor estão contidos na Tabela 2. 

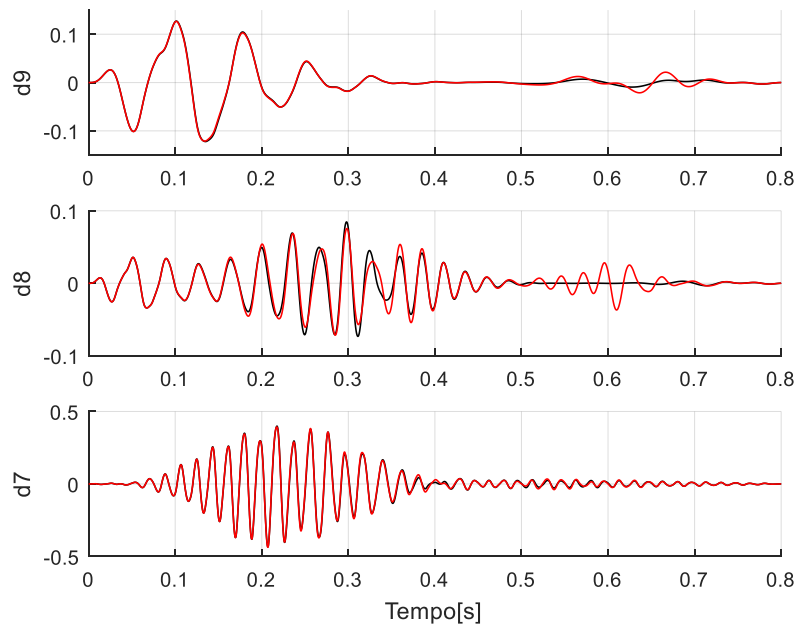

Figura 4 - Sinais de corrente decompostos para condição de rotor saudável (curva preta) e com uma barra quebrada (curva vermelha)
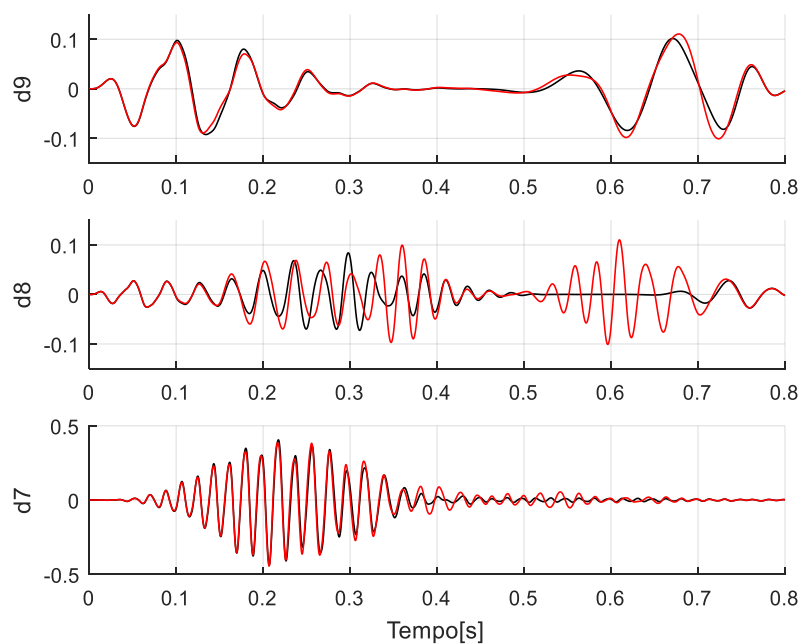

Figura 5 - Sinais de corrente decompostos para condição de rotor saudável (curva preta) e com duas barras quebradas (curva vermelha)

Tabela 2 - Níveis de Energia para as três condições de Operação do Rotor

\begin{tabular}{|c|c|c|c|}
\hline & $\begin{array}{c}\text { Rotor } \\
\text { Saudável }\end{array}$ & $\begin{array}{c}\text { 1 Barra } \\
\text { Quebrada }\end{array}$ & $\begin{array}{c}\text { 2 Barras } \\
\text { Quebradas }\end{array}$ \\
\hline D7 & 0.755732 & 0.742707 & 0.706934 \\
\hline D8 & 0.029869 & 0.031661 & 0.076717 \\
\hline D9 & 0.074804 & 0.089725 & 0.090939 \\
\hline
\end{tabular}

\section{B. Motor de $500 \mathrm{HP}$}

Aplicando os mesmos critérios de decomposição para Transformada de Wavelet no motor de 500 HP também é possível verificar a influência da falha de barra quebrada através do sinal de corrente, independente da sua magnitude e da potência fornecida pelo motor. As diferenças entre os dois motores aparecem no tempo de partida da máquina. A potência maior do segundo motor acarreta em um período transitório mais extenso, facilitando a deteç̧ão do problema pela decomposição via Wavelet. As Figuras 6 e 7 apresentam os resultados para condição saudável, comparando com uma e duas barras quebradas, respectivamente.
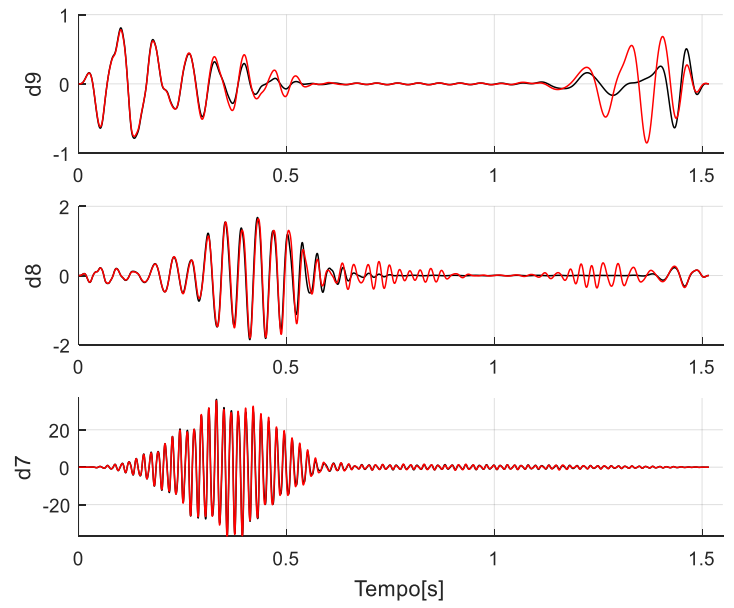

Figura 6 - Sinais de corrente decompostos para condição de rotor saudável (curva preta) e com uma barra quebrada (curva vermelha)
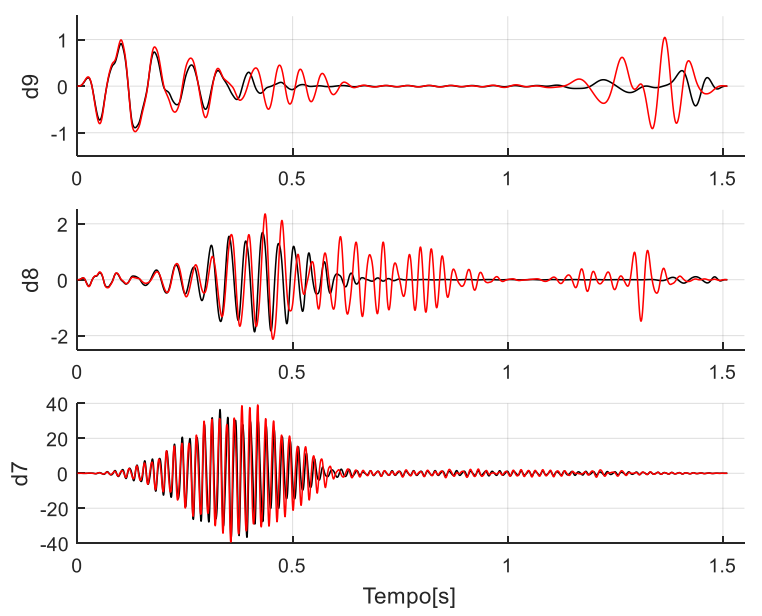

Figura 7 - Sinais de corrente decompostos para condição de rotor saudável (curva preta) e com duas barras quebradas (curva vermelha)

Novamente, através do cálculo dos níveis de energia para os coeficientes da Transformada de Wavelet é possível quantificar e comparar a influência das barras quebradas sobre o sinal da corrente de partida. A Tabela 3 apresenta os níveis de energia para as três condições de operação do rotor.

Tabela 3 - Níveis de Energia para as três condições de Operação do Rotor

\begin{tabular}{|c|c|c|c|}
\hline & $\begin{array}{c}\text { Rotor } \\
\text { Saudável }\end{array}$ & $\begin{array}{c}\text { 1 Barra } \\
\text { Quebrada }\end{array}$ & $\begin{array}{c}\text { 2 Barras } \\
\text { Quebradas }\end{array}$ \\
\hline D7 & 0.941690 & 0.915807 & 0.922039 \\
\hline D8 & 0.002732 & 0.003516 & 0.008529 \\
\hline D9 & 0.007378 & 0.034409 & 0.025989 \\
\hline
\end{tabular}

\subsection{Resultado experimental-Motor $3 \mathrm{CV}$}

Através dos dados de corrente de alimentação extraídos do motor foi possível encontrar os coeficientes da WT para condição de rotor saudável e com a presença de uma barra quebrada. A Figura 8 apresenta a comparação entre o sinal de corrente para as duas condições considerando os níveis 7,8 e 9, visto que correspondem aos intervalos de frequência em que a falha de barras quebradas pode se manifestar. 

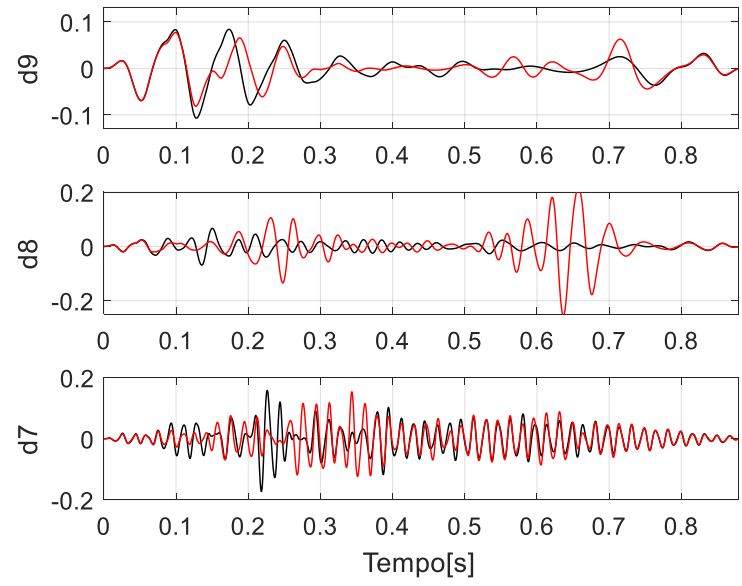

Figura 8 - Sinais de corrente decompostos para condição de rotor saudável (curva preta) e com uma barra quebrada (curva vermelha)

Para a análise experimental foram avaliadas a condição de rotor saudável e com uma barra quebrada. Essa condição representa o estágio inicial da falha, e por isso, é mais difícil de ser identificada. A Tabela 4 mostra a comparação entre duas situações analisadas para o rotor.

Tabela 4 - Níveis de Energia para as duas Condições de Operação do Rotor

\begin{tabular}{|c|c|c|}
\hline & Rotor Saudável & 1 Barra Quebrada \\
\hline D7 & 0.102350 & 0.271412 \\
\hline D8 & 0.017141 & 0.173532 \\
\hline D9 & 0.014214 & 0.052784 \\
\hline
\end{tabular}

Durante a partida do motor de indução trifásico, a frequência correspondente a falha no rotor varia de acordo com o escorregamento da máquina, e por isso, a necessidade de avaliar mais de um nível de decomposição na transformada de Wavelet.

As três condições de operação apresentadas demonstram uma característica comum e que pode ser usada para a detecção da falha de barras quebradas. As variações encontradas nos níveis de energia sugerem alterações nos sinais de corrente provenientes da presença de uma ou mais barras quebradas. Quando comparado ao rotor saudável, verifica-se que os níveis de energia sofrem variações, que também podem ser visualizados através dos gráficos com a decomposição dos sinais em níveis de frequência.

Para os gráficos obtidos através das simulações nota-se um aumento do nível de energia em D8 e D9, enquanto D7 sofre uma redução. No caso dos testes experimentais, D8 e D9 também apresentaram um acréscimo, seguido pelo coeficiente D7. Essas variações se tornam mais acentuadas e podem ser mais facilmente percebidas nos gráficos de acordo com o aumento do número de barras quebradas. Ou seja, esse aumento acentua a influência da falha no rotor sobre o sinal de corrente extraído da alimentação do motor.

Os resultados das simulações anteriores mostram as conclusões obtidas utilizando os valores de corrente em regime transitório, obtidos durante o processo de partida do motor. Sabe-se que o diagnóstico das falhas de barras quebradas pode ser realizado através das frequências de bandas laterais e que devido a variação do escorregamento durante a partida, esses valores não são fixos, ficando concentrados em intervalos como os analisados anteriormente.

Como forma de embasar a metodologia apresentada para o regime transitório, os dados da máquina em regime permanente foram também analisados através da transformada de Wavelet juntamente com a extração do envelope através da Transformada de Hilbert. Nesse caso, como a máquina opera com velocidade constante, sabe-se exatamente qual o valor da frequência de barra quebrada. A máquina utilizada para compor esses resultados foi simulada com $80 \%$ de carga no eixo (ou 9,5 N.m), produzindo um escorregamento de 0,031. Dessa forma, pode-se afirmar que a frequência de barra quebrada vale aproximadamente $63,7 \mathrm{~Hz}$, ou $3,7 \mathrm{~Hz}$ considerando o sinal extraído pela $\mathrm{HT}$, devendo estar localizada no nível D10 (entre 2,5 e $5 \mathrm{~Hz}$ ) da decomposição de Wavelet.

Aplicando os conceitos da Transformada de Wavelet no sinal extraído através da Transformada de Hilbert, obtêm-se o seguinte sinal decomposto para o nível D10, apresentado pela Figura 10.

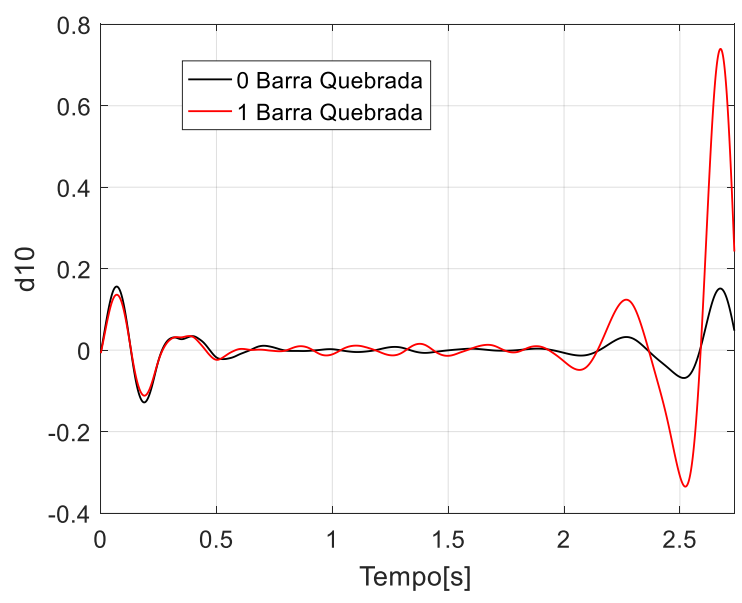

Figura 10 - Sinal decomposto via WT para o nível D10

A Tabela 5 apresenta os valores para os níveis de energia considerando numericamente a variação expressa na Figura 10 e a diferença percentual entre o valor de energia para a condição de rotor saudável e com uma barra quebrada.

Tabela 5 - Níveis de Energia para as duas Condições de Operação do Rotor

\begin{tabular}{|c|c|c|c|}
\hline & $\begin{array}{c}\text { Rotor } \\
\text { Saudável }\end{array}$ & $\begin{array}{c}\text { 1 Barra } \\
\text { Quebrada }\end{array}$ & $\begin{array}{c}\text { Variação } \\
\text { Percentual }\end{array}$ \\
\hline D10 & 0.079492 & 0.248539 & 312.658234 \\
\hline
\end{tabular}

Assim como foi relatado anteriormente, sabe-se que frequência de barra quebrada, calculada através da Equação 5, está inserida no intervalo abrangido pelo coeficiente D10. Os valores apresentados na Tabela 5, juntamente com o gráfico presente na Figura 10, comprovam o diagnostico apresentado para a máquina através da utilização do sinal em regime transitório. 


\section{CONLUSÕES}

A detecção de falhas de barras quebradas através da metodologia apresentada obteve resultados consideráveis e demonstraram a possibilidade de utilizar a corrente de partida do motor para chegar em uma determinada conclusão sobre o estado de funcionamento do rotor. Os métodos propostos são relativamente simples e não necessitam de testes invasivos na máquina, podendo ser realizados com o motor em funcionamento. Atualmente, a maioria das aplicações industriais permite a obtenção de tais correntes de forma remota através de sistemas supervisórios, e em muitas aplicações, a máquina opera apenas em pequenos intervalos de tempo, dificultando a análise em regime permanente. Além disso, a técnica proposta não depende da carga. A falha pode ser até diagnosticada com operação da máquina na condição com carga reduzida.

A semelhança de comportamento dos resultados apresentados e o prévio conhecimento do estado do rotor permitem afirmar que o método utilizado em regime transitório cumpriu o propósito de detectar a presença de uma falha na máquina. Além do mais, o conceito de Energia da Wavelet permite tecer informações a respeito da gravidade do problema. É possível criar uma assinatura da máquina, e com isso, acompanhar a evolução da falha.

O resultado em regime permanente apresentado foi utilizado para comparar com as análises feitas em regime transitório e para demonstrar o comportamento da frequência da falha nas condições de partida com escorregamento variável e durante operação com carga e escorregamento constante.

A análise da corrente de partida através da WT, apresentado nesse trabalho, surge como uma alternativa aos métodos de detecção de falhas utilizando os sinais da máquina obtidos em regime permanente, preenchendo essa lacuna existente nos estudos de detecção de falhas.

\section{AGRADECIMENTOS}

Os autores agradecem à CAPES/CNPq/FAPEMIG/Inerge e à UFSJ pelos auxílios financeiros recebidos.

\section{REFERÊNCIAS}

Ahamed, S. K. et al. Novel Diagnosis Technique of Mass Unbalance in Rotor of Induction Motor by the Analysis of Motor Starting Current at No Load Through Wavelet Transform. International Conference on Electrical and Computer Engineering, 2010, Dhaka. Dhaka: ICECE, 2010, v.1, p. $474-477$.

Alves, D. A. Técnicas de Detecção de Falhas em Barras do Rotor nos Motores de Indução Trifásicos. Dissertação de Mestrado, PPGEL - Programa de Pós-Graduação em Engenharia Elétrica da UFSJ. 2017.

Avelar, V. S., Baccarini, L. M. R. e Amaral, G. F. V. Desenvolvimento de um sistema inteligente para diagnóstico de falhas nos enrolamentos do estator de motores de indução. X SBAI - Simpósio Brasileiro de Automação Inteligente, 2011.
Baccarini, L. M. R., Menezes, B. M., Caminhas, W. M. Fault induction dynamic model, suitable for computer simulation: Simulation results and experimental validation, Mechanical Systems and Signal Processing, Volume 24, Issue 1, 2010, Pages 300-311, ISSN 08883270.

Baccarini, L. M. R. Detecção e Diagnósticos de Falhas em Motores de Indução. Tese de Doutorado - Universidade Federal de Minas Gerais, Belo Horizonte, 2005.

Bidgoli, F. S.; Poshtan, J. Application of improved HilbertHuang and wavelet packet transforms in broken rotor bar fault detection. Power Electronics, Drive Systems \& Technologies Conference (PEDSTC), v. 8, 2017, Mashhad. Proceedings... Mashhad: PEDSTC, 2017, p. 341-346.

Hernández, J. C. et al., Comparison of Different Wavelet Families for Broken Bar Detection in Induction Motors. IEEE - International Conference on Industrial Technology (ICIT), 2015, Seville. Proceedings... Seville: ICIT, 2015, P. 3220-3225.

Krause, P. C., Wasynczuk, O. and Sudhof, S. D. (2002). Analysis of Electric Machinery and Drive Systems, Academic Press - New Jersey. IEEE Transactions on Power Systems, Vol. 9, No. 4, pp. 1942- 1948.

Liu, T., e Huang, J. (2005). A novel method for induction motors stator interturn short circuit fault diagnosis by wavelet packet analysis. IEEE Transactions on Electrical Machines and Systems, 3(1):2254-2258.

Massirer, D. A., Sistema especialista protótipo para auxílio à verificação da estrutura de produto de motores elétricos. Universidade Federal de Santa Catarina, 2007.

Nóbrega Sobrinho, A. C., Lima Filho, A. C., Sena, A. P. C., Brito, J. N., Silva, J. F., Mafaldo, Í. M., Transformada Wavelet para detecção de barras quebradas em Motores de Indução Trifásicos. Revista Principia - Divulgação Científica e Tecnológica do IFPB. 1. 72.

Pineda-Sanchez, M., Riera-Guasp, M., Roger-Folch, J., Antonino-Daviu, J. A., Perez-Cruz, J. e Puche-Panadero, R. "Diagnosis of Induction Motor Faults in TimeVarying Conditions Using the Polynomial-Phase Transform of the Current,". IEEE Transactions on Industrial Electronics, vol. 58, no. 4, pp. 1428-1439, April 2011.

Riera-Guasp, M., Pineda-Sanchez, M., Perez-Cruz, J., PuchePanadero, R., Roger-Folch, J. e Antonino-Daviu, J. A., "Diagnosis of Induction Motor Faults via Gabor Analysis of the Current in Transient Regime," in IEEE Transactions on Instrumentation and Measurement, vol. 61, no. 6, pp. 1583-1596, June 2012.

Sridhar, S., Rao, K. U., Jade, S. Detection of Broken Rotor Bar Fault in Induction Motor at Various Load Conditions Using Wavelet Transforms. International Conference on Recent Developments in Control, Automation and Power Engineering (Rdcape), 2015, Noida. Proceedings. Noida: Rdcape, 2015, P. 77-82.

Zhang, P., Du, Y., Habetler, T. G. e Lu, B. "A survey of condition monitoring and protection methods for medium-voltage induction motors," IEEE Trans. Ind. Appl., vol. 47, no. 1, pp. 34-46, Jan./Feb. 2011. 